\title{
Improved procedure on the microscopic approach to determine colloidal stability
}

\author{
Zhiwei Sun ${ }^{\text {a),b) }}$ \\ Hefei National Laboratory for Physical Sciences at Microscale and Physics Department, University of \\ Science and Technology of China, Hefei, Anhui 230026, People's Republic of China and National \\ Microgravity Laboratory (NML), Institute of Mechanics, Chinese Academy of Sciences, \\ Beijing 100080, People's Republic of China \\ Shenghua $\mathrm{Xu}$ \\ Hefei National Laboratory for Physical Sciences at Microscale and Physics Department, \\ University of Science and Technology of China, Hefei, Anhui 230026, People's Republic of China \\ Jie Liu \\ National Microgravity Laboratory (NML), Institute of Mechanics, Chinese Academy of Sciences, \\ Beijing 100080, People's Republic of China \\ Yinmei $\mathrm{Li}^{\mathrm{a}), \mathrm{c})}$ and Liren Lou \\ Hefei National Laboratory for Physical Sciences at Microscale and Physics Department, \\ University of Science and Technology of China, Hefei, Anhui 230026, People's Republic of China \\ Jingchang Xie \\ National Microgravity Laboratory (NML), Institute of Mechanics, Chinese Academy of Sciences, \\ Beijing 100080, People's Republic of China
}

(Received 5 January 2005; accepted 21 February 2005; published online 9 May 2005)

\begin{abstract}
We present an improved procedure on the approach to determine the stability of polystyrene spheres at microscopic particle levels by means of artificially induced particle collisions with the aid of optical tweezers [J. Chem. Phys. 119, 2399 (2003)]. The basic consideration on this new development is that the major contribution to the sticking probability for a particle pair caught into the optical trap for a short period is from its single collision; therefore, if the trapping duration for the pair is taken to be short, the accumulated sticking probability will be a good approximation for the single collision. The experimental procedure associated with this approximation does not resort to exactly controlling the short trapping duration or request the trapping duration correction as previously reported, and therefore it is more practical and applicable for a broader range of the stability ratio. The experimental results under different electrolyte concentrations by the new procedure are consistent with those from the turbidity measurements. (C) 2005 American Institute of Physics. [DOI: 10.1063/1.1890926]
\end{abstract}

\section{INTRODUCTION}

In the previous paper, ${ }^{1}$ we reported a method to determine the stability of polystyrene suspensions by means of artificially induced particle collisions with the aid of optical tweezers. Because the method needs to correct undesired multicollisions during the trapping duration, for each experiment condition two accumulated sticking probabilities with different trapping durations $(\tau)$ are required. The relevant procedure not only involves much more tedious experimental endeavor, but also restricts its applicable range because the measurement of two accumulated sticking probabilities becomes impracticable when the sticking probability is close to one. An improved procedure to overcome this shortcoming and extend the applicable range is presented in this paper.

\footnotetext{
a) Authors to whom correspondence should be addressed.

${ }^{b)}$ Present address: NML, Institute of Mechanics, Chinese Academy of Sciences, Beijing 100080, P. R. China. Electronic mail: sunzw@imech.ac.cn

${ }^{c)}$ Present address: Physics Department, University of Science and Technology of China, Hefei, Anhui 230026, P. R. China. Electronic mail: liyinmei@ustc.edu.cn
}

\section{MODEL ANALYSIS}

Let $P(\tau)$ be the accumulated sticking probability for a particle pair caught into the optical trap at the time $t=0$ and released at the time $t=\tau$, and let $p$ be the sticking probability of a single collision. Following the analyses and assumption in Ref. 1, the particle pair experiences "compact" and "relaxed" statuses, and we have

$$
P(\tau)=p f_{c} \tau_{1}+p f_{r}\left(\tau-\tau_{1}\right),
$$

where $\tau_{1}$ is the duration for which the compact status lasts, and $f_{c}$ and $f_{r}$ are the collision frequencies in the compact and relaxed statuses, respectively.

Because $f_{c} \gg f_{r}$, as shown in Ref. 2, and using the assumption that only a single collision takes place for a particle pair in the compact status we have $f_{c} \tau_{1}=1$. The following equation is suggested ${ }^{1}$ to evaluate $p$ when $\tau \gg \tau_{1}$ :

$$
p \approx P(\tau)-p f_{r} \tau
$$

After measuring two sets of $P$ at different $\tau\left(\tau^{\prime}\right.$ and $\left.\tau^{\prime \prime}\right)$, $p f_{r}$ can be calculated, $p f_{r}=\left(P\left(\tau^{\prime}\right)-P\left(\tau^{\prime \prime}\right)\right) /\left(\tau^{\prime}-\tau^{\prime \prime}\right)$. The 
physical meaning of Eq. (2) is that the accumulated sticking probability $P(\tau)$ during the trapping duration contains the contributions of both single collision (in the compact status) and multicollisions (in the relaxed status), so the latter (the second term) must be subtracted from $P(\tau)$.

All the data of the sticking probability and stability ratio through the microscopic approach presented in Ref. 1 are obtained by using Eq. (2) with $\tau^{\prime}=1 \mathrm{~s}$ and $\tau^{\prime \prime}=2 \mathrm{~s}$. The major disadvantage in this strategy is that for each experimental condition, two accumulated sticking probabilities $P(\tau)$ with different trapping durations $\left(\tau^{\prime}\right.$ and $\left.\tau^{\prime \prime}\right)$ are required, therefore making the measurements less practical. In addition, its applicable range is restricted because it is impossible to measure two $P(\tau)$ when $p$ is close to one.

Now we consider the possibility to simplify and improve the above procedure. The improvement is based on the following consideration. If our assumption that one and only one collision takes place for the particle pair in the compact status holds, considering the condition $f_{c} \gg f_{r}$, it is possible to find a reasonable time duration $\tau$, in which the primary contribution to $P(\tau)$ is from $p f_{c} \tau_{1}$ due to the collision in the compact status and that the contribution from the collision in the relaxed status is small. Actually, as long as $t$ is long enough to cover $t_{1}$ and short enough to ensure $\left(\tau-\tau_{1}\right) \ll 1$, the contribution from $p f_{r} \tau$ should be so small that $P(\tau)$ $\approx p f_{c} \tau_{1} \approx p$. That is,

$$
p \approx P(\tau) \text { when } \tau_{1}<\tau \ll 1 .
$$

Ideally, to minimize the contribution of the multicollisions we should choose $\tau$ as short as possible. In practicing, however, it is hard to visually determine the exact moment when two particles start to be held by the optical tweezers simultaneously. Besides, the particle pair trapped in the optical tweezers is lined up in the $z$ direction (along laser beam); that is, in the image we view, one particle is behind the other. This spatial configuration presents an additional difficulty to determine the moment when two particles are actually captured into the trap, and therefore have no way to control the exact time to release them for a predetermined trapping duration. However, it is not difficult to meet the condition of Eq. (3). For instance, if a particle pair is held in the trap for $0.2 \mathrm{~s}$, it should have plenty of time to complete its collision once in the compact status; also, $0.2 \mathrm{~s}$ is still short enough to make the term $p f_{r} \tau$ reasonably small. As a matter of fact, it can be expected that the uncertainty of the trapping duration would not significantly alter the measurements made using Eq. (3).

We call the sticking probability $p$, evaluated by Eq. (3), "short-trapping approximation," which is drawn from the above discussion as a deduction of the physical model described in Ref. 1. The correctness of Eq. (3) has been verified by the results of relevant experiments described below.

\section{EXPERIMENT}

The experiment setup used in our experiment is the same as that in Ref. 1. Because the particles used in the experiment of Ref. 1, polystyrene (PS) spheres with a radius of $a_{0}$ $=0.497 \pm 0.011 \mu \mathrm{m}$ (4009A from Duke Scientific Corpora- tion, USA), have passed their expiration date (May 2003), in the present study fresh particles (also 4009A from Duke, the expiration date: August 2006) were employed. We found their characteristics are rather different from that used in Ref. 1. We suppose the aging and storage experience may cause this difference. Considering this fact, in this study the fresh samples were used in the turbidity measurements and experiments with the optical tweezers.

The experimental procedure to confine a particle pair in the optical trap for collisions is basically the same as that described in Ref. 1. The only difference is making the trapping duration short enough to meet the requirement of Eq. (3). To do so, after the second particle was captured into the trap (while the first one was already in the trap), we released them after a very short delay (to cover $\tau_{1}$ ) to ensure a collision to complete and then traced them to check if they remained together or separated. The above procedure was manually operated, and the starting point of the time of a trapping duration is determined only visually, so it is difficult to accurately control the length of each artificial collision for a predetermined trapping duration. Our estimate of the corresponding trapping duration used in the present experiment is about $0.2( \pm 0.05) \mathrm{s}$, on average.

Considering that a longer trapping duration will bring more contributions from multicollision, and therefore causes more error into the measurement (make the sticking probability too high), we should choose the trapping duration as short as possible. In practice, however, we found that too short trapping duration made the data scattered. We assume that this uncertainty in data is associated with the error to determine visually the very beginning $(\tau=0)$ for two particles captured into the trap. The fact that one particle is behind the other in the trap further reduces our visual resolution capacity to judge the time of $\tau=0$ with accuracy. In this case, sometimes we may release the particle pair actually before they come together for collision, and therefore count some pairs that have not collided as "have collided" into the statistics, causing the uncertainties. Apparently, the reproducibility of the data should be the first important factor for the measurement of the sticking probability. Therefore $t$ should be chosen appropriately to ensure that the data is stable, and that the trapping duration is short enough. We found that $0.2 \mathrm{~s}$ is about the shortest duration to keep the data stable, although it is still too long to completely avoid the influence of the multicollision, as discussed below.

To obtain the sticking probability $P$, we needed to perform a series of the aforementioned collision tests. Each time we tested one particle pair for their collision outcome. After sampling $n$ pairs of particles for such artificial collisions, using Eq. (3) under the "short-trapping approximation," we could directly evaluate the sticking probability by calculating the ratio of the number of sticking pairs $n_{c}$ to $n$.

To compare the stability ratios $W$ obtained from the above microscopic approach with those from the macroscopic approach (denoted by $W_{\text {macro }}$ ), we also did turbidity measurements $^{1,3}$ for the new PS samples with ultraviolet/ visible spectrophotometer (UV-2100, BRAIC, China) and the zeta potentials. 
TABLE I. Sticking probability $(P)$, stability ratio $(W)$, and zeta potential vs concentrations of $\mathrm{NaCl}\left(C_{\mathrm{NaCl}}\right)$.

\begin{tabular}{llllllll}
\hline \hline$C_{\mathrm{NaCl}}\left(\mathrm{mol} \mathrm{L}^{-1}\right)$ & 0 & 0.05 & 0.1 & 0.15 & 0.20 & 0.25 & 0.3 \\
$p_{\text {micro }}(\%)$ & 0 & $2.3( \pm 0.9)$ & $11( \pm 2)$ & $32( \pm 3)$ & $63( \pm 5)$ & $93( \pm 6)$ & 100 \\
$p_{\text {macro }}(\%)$ & 0 & $3( \pm 2)$ & $11( \pm 3)$ & $27( \pm 4)$ & $59( \pm 6)$ & $90( \pm 4)$ & 100 \\
$W_{\text {micro }}$ & $\sim \infty$ & $43( \pm 17)$ & $9.1( \pm 1.7)$ & $3.1( \pm 0.3)$ & $1.59( \pm 0.13)$ & $1.08( \pm 0.07)$ & 1.00 \\
$W_{\text {macro }}$ & $\sim \infty$ & $33( \pm 22)$ & $9.1( \pm 2.5)$ & $3.7( \pm 0.5)$ & $1.69( \pm 0.17)$ & $1.11( \pm 0.05)$ & 1.00 \\
Zeta potential $(\mathrm{mv})$ & $-57( \pm 2)$ & $-55( \pm 2)$ & $-50( \pm 2)$ & $-38( \pm 2)$ & $-32( \pm 4)$ & $-29( \pm 4)$ & $-26( \pm 4)$ \\
\hline \hline
\end{tabular}

$\overline{\text { All experiments were performed at } 25^{\circ} \mathrm{C} . p_{\text {micro }} \text { and } W_{\text {micro }} \text { are obtained through the microscopic approach }}$ based on Eq. (3); $p_{\text {macro }}$ and $W_{\text {macro }}$ are from the macrosocpic approach by the turbidity measurements.

\section{RESULTS AND DISCUSSION}

All data obtained through the microscopic approach under the short-trapping approximation [Eq. (3)] and those from the macroscopic approach by the turbidity measurements ( $W_{\text {macro }}$ is the stability ratio and $p_{\text {macro }}=1 / W_{\text {macro }}$ is the sticking probability), as well as zeta potentials are listed in Table I. We can see that for the whole range of the concentration of $\mathrm{NaCl}$ (up to $0.5 \mathrm{~mol} \mathrm{~L}^{-1}$ ), namely, from the stable to the rapid-coagulated status of the polystyrene suspensions, the differences between the results from the microscopic approach and those from the macroscopic approach are basically within the limits of the error in the measurements.

However, the experimental data seem to show, to some extent, a gradual transition from $p_{\text {micro }} \leqslant p_{\text {macro }}$ to $p_{\text {micro }}>p_{\text {macro }}$ with increasing electrolyte concentration. Our explanation for this transition is that the contribution of $p f_{r}\left(\tau-\tau_{1}\right)$ (the multicollision contribution), in Eq. (3), to $p_{\text {micro }}$ becomes large because both $p$ and $f_{r}$ become larger when the repulsion between particles is reduced at higher electrolyte concentrations. Therefore as long as $\left(\tau-\tau_{1}\right)>0$, the multicollision effect will be growing with decreasing repulsion between particles. This statement has been verified by the experimental fact shown in Table II. In the $C_{\mathrm{NaCl}}$ $=0.1 \mathrm{~mol} \mathrm{~L}^{-1}$ case, the contribution to the accumulated sticking probability $P$ from the collisions occurring from 0.2 to $1 \mathrm{~s}$ is $5 \%$, but when $C_{\mathrm{NaCl}}$ was increased to $0.15 \mathrm{~mol} \mathrm{~L}^{-1}$, the relevant contribution becomes $17 \%$. We can also see that the values of $p_{\text {micro }}$, evaluated by using the short-trapping approximation of Eq. (3) and the multicollision correction of Eq. (2), are very close. But for the former, it needs to measure only one quantity, $P(\tau \approx 0.2 \mathrm{~s})$, and for the latter, two quantities, $P\left(\tau^{\prime}\right)$ and $P\left(\tau^{\prime \prime}\right)$, are required to be measured.

The obvious superiority of the short-trapping approximation of Eq. (3) to the multicollision correction formula of Eq. (2) is its broader applicable range. Equation (3) is applicable to almost the whole range up to the critical coagulation concentration of $\mathrm{NaCl}$, but Eq. (2) is only effective for concentrations of $\mathrm{NaCl}$ less than $0.2 \mathrm{~mol} \mathrm{~L}^{-1}$.

The experimental results which coincide with the modeling prediction provide direct evidence for the hypothesis that there will be, approximately, only one collision for the particles in the short trapping duration. Taking the experimental data at $C_{\mathrm{NaCl}}=0.1 \mathrm{~mol} \mathrm{~L}^{-1}$ as an example, the accumulated sticking probability $P$ within the first $0.2 \mathrm{~s}$ has reached $11 \%$, which is very close to that from the turbidity measurement, while the contribution to $P$ from the successive $0.8 \mathrm{~s}$ is only $4 \%$. These data imply that (1) the collision frequency within the first $0.2 \mathrm{~s}$ is much higher than that afterwards and that (2) at least one and only one collision takes place within the first $0.2 \mathrm{~s}$. Otherwise, $P$ would have had a much larger fluctuation and would not have been so close to that from the turbidity measurement due to a lack of accuracy in controlling the trapping duration of $0.2 \mathrm{~s}$.

When the potential barrier $V_{\max }$, resulting from the combination of attractive and repulsive interactions, is higher than the particle's average thermal energy $k T$, the sticking probabilities are small. It has been shown ${ }^{1}$ that the trapping speed has little effect on the observed sticking probabilities at lower electrolyte $(\mathrm{NaCl})$ concentrations $\left(C_{\mathrm{NaCl}}\right.$ $\left.=0.1 \mathrm{~mol} \mathrm{~L}^{-1}\right)$. The short-trapping procedure in this study is able to measure the sticking probability at higher electrolyte concentrations, at that condition the potential barrier is lower and the laser intensity (associated with the trap strength or trapping speeds) may become significant in the sticking probability measurement. From Table I we can see that $p_{\text {micro }}=63( \pm 5) \%$ when $C_{\mathrm{NaCl}}=0.2 \mathrm{~mol} \mathrm{~L}^{-1}$. When we raised the laser power from 18.7 to $66 \mathrm{~mW}$, there was an increase of $10 \%$ in the $p_{\text {micro }}$ observed. Apparently, this trapping strength effect is related to the relative magnitudes of the barrier and the thermal velocity of particles. To minimize this influence in the practice, we choose the trapping strength as low as possible.

\section{CONCLUSION}

The relevant experiments have confirmed our conjecture (short-trapping approximation) that the sticking probability for two particles held in the optical trap for a short period (about $0.2 \mathrm{~s}$ ) is approximately equal to the commonly referred sticking probability. The sticking probabilities or the stability ratios measured through this simplified microscopic

TABLE II. Comparison of sticking probabilities $\left(p_{\text {micro }}\right)$, evaluated according to Eq. (2) with $\tau^{\prime}=1 \mathrm{~s}$ and $\tau^{\prime \prime}$ $=2 \mathrm{~s}$ and Eq. (3).

\begin{tabular}{lcccc}
\hline \hline$C_{\mathrm{NaCl}}\left(\mathrm{mol} \mathrm{L}^{-1}\right)$ & $p_{\text {micro }} \approx P(\tau \approx 0.2 \mathrm{~s})$ & $P(\tau=1 \mathrm{~s})$ & $P(\tau=2 \mathrm{~s})$ & $p_{\text {micro }} \approx 2 P(\tau=1 \mathrm{~s})-P(\tau=2 \mathrm{~s})$ \\
\hline 0.1 & $11 \%$ & $16 \%$ & $20 \%$ & $12 \%$ \\
0.15 & $32 \%$ & $49 \%$ & $67 \%$ & $31 \%$ \\
\hline \hline
\end{tabular}


approach are consistent with those from the turbidity measurements, basically for the whole range of the electrolyte $\left(C_{\mathrm{NaCl}}\right)$ concentrations from 0 to $0.3 \mathrm{~mol} \mathrm{~L}^{-1}$.

Compared with the approach originally reported in Ref. 1 , the present method is more practicable because of its simplicity and broader applicable range.

Our experiments showed that the effect of trapping strength becomes noticeable when the potential barrier is lower, suggesting the trapping strength should be chosen as low as possible.

Our modeling analysis and the associated experiment provide useful information for gaining insight into the collision-reaction process of the two-particle system confined in an optical trap.

\section{ACKNOWLEDGMENTS}

This work is supported by Grant No. 20273065, No. 20473108 from the National Natural Science Foundation of China and the "Knowledge Innovation Program" of the Chinese Academy of Sciences.

${ }^{1}$ Z. W. Sun, S. H. Xu, G. L. Dai, Y. M. Li, L. R. Lou, Q. S. Liu, and R. Z. Zhu, J. Chem. Phys. 119, 2399 (2003).

${ }^{2}$ Z. W. Sun, R. Z. Zhu, Y. M. Li, S. H. Xu, and L. R. Lou, Acta PhysicoChimica Sinica 19, 1078 (2003).

${ }^{3}$ Z. W. Sun and R. L. Qiao, J. Colloid Interface Sci. 223, 126 (2000). 